\title{
Correction to: Early central nervous system relapse of monomorphic epitheliotropic intestinal T-cell lymphoma after cord blood transplantation
}

\author{
Yuma Nato $^{1,2} \cdot$ Kana Miyazaki $^{1}(1) \cdot$ Hiroshi Imai $^{3} \cdot$ Eri Nakano $^{1} \cdot$ Yuki Kageyama $^{1} \cdot$ Kazuko Ino $^{1} \cdot$ Atsushi Fujieda $^{1,4}$. \\ Takeshi Matsumoto $^{1,5} \cdot$ Isao Tawara $^{1} \cdot$ Kyosuke Tanaka $^{6} \cdot$ Motoko Yamaguchi $^{1} \cdot$ Naoyuki Katayama $^{1,7}$
}

Published online: 25 January 2022

(c) Japanese Society of Hematology 2022

\section{Correction to: \\ International Journal of Hematology (2021) 114:129-135 https://doi.org/10.1007/s12185-021-03107-9}

In the original publication of the article, the Table 1 was published with errors. The correct Table 1 is given in this correction.
Publisher's Note Springer Nature remains neutral with regard to jurisdictional claims in published maps and institutional affiliations.

The original article can be found online at https://doi.org/10.1007/ s12185-021-03107-9.

Kana Miyazaki

kmiyazaki@clin.medic.mie-u.ac.jp

1 Department of Hematology and Oncology, Mie University Graduate School of Medicine, 2-174 Edobashi, Tsu, Mie 514-8507, Japan

2 Department of Hematology, Yokkaichi Municipal Hospital, Yokkaichi, Japan

3 Pathology Division, Mie University Hospital, Tsu, Japan

4 Department of Hematology, Japanese Red Cross Ise Hospital, Ise, Japan

5 Department of Transfusion Medicine and Cell Therapy, Mie University Hospital, Tsu, Japan

6 Department of Endoscopy, Mie University Hospital, Tsu, Japan

7 Suzuka University of Medical Science, Suzuka, Japan 
Table 1 Laboratory data on admission

\begin{tabular}{|c|c|c|c|c|c|}
\hline \multicolumn{2}{|c|}{ Complete blood count } & \multicolumn{2}{|c|}{ Serum biochemistry } & \multicolumn{2}{|l|}{ Serological test } \\
\hline WBC & $8440 / \mu \mathrm{L}$ & $\mathrm{TP}$ & $6.3 \mathrm{~g} / \mathrm{dL}$ & CRP & $2.30 \mathrm{mg} / \mathrm{dL}$ \\
\hline Neutro & $82.8 \%$ & Alb & $3.7 \mathrm{~g} / \mathrm{dL}$ & IgG & $818 \mathrm{mg} / \mathrm{dL}$ \\
\hline Lym & $7.6 \%$ & BUN & $8.9 \mathrm{mg} / \mathrm{dL}$ & $\operatorname{Ig} \mathrm{A}$ & $269 \mathrm{mg} / \mathrm{dL}$ \\
\hline Mono & $9.4 \%$ & $\mathrm{Cr}$ & $0.35 \mathrm{mg} / \mathrm{dL}$ & $\operatorname{IgM}$ & $96 \mathrm{mg} / \mathrm{dL}$ \\
\hline Baso & $0.1 \%$ & UA & $7.9 \mathrm{mg} / \mathrm{dL}$ & EB/EA-IgG & $<10$ \\
\hline Eosino & $0.1 \%$ & AST & $19 \mathrm{IU} / \mathrm{L}$ & EB/EA-IgM & $<10$ \\
\hline $\mathrm{RBC}$ & $425 \times 10^{4} / \mu \mathrm{L}$ & ALT & $16 \mathrm{IU} / \mathrm{L}$ & EB/EBNA & 80 \\
\hline $\mathrm{Hb}$ & $12.5 \mathrm{~g} / \mathrm{dL}$ & ALP & $136 \mathrm{IU} / \mathrm{L}$ & EB.VCA-IgG & 80 \\
\hline $\mathrm{Ht}$ & $37.1 \%$ & LDH & $188 \mathrm{IU} / \mathrm{L}$ & EB.VCA-IgM & $<10$ \\
\hline $\mathrm{MCV}$ & $87.3 \mathrm{fL}$ & T-Bil & $0.7 \mathrm{mg} / \mathrm{dL}$ & & \\
\hline $\mathrm{MCH}$ & $29.4 \mathrm{pg}$ & SIL-2R & $1938 \mathrm{U} / \mathrm{mL}$ & Blood coagulation & \\
\hline $\mathrm{MCHC}$ & $33.7 \mathrm{~g} / \mathrm{dL}$ & $\mathrm{HbA} 1 \mathrm{c}$ & $5.3 \%$ & APTT & $26.5 \mathrm{sec}$ \\
\hline Plt & $540 \times 10^{3} / \mu \mathrm{L}$ & & & PT-INR & 1.10 \\
\hline
\end{tabular}

